\title{
Magnetic structure and magnon dynamics of the quasi-two-dimensional antiferromagnet $\mathrm{FePS}_{3}$
}

\author{
D. Lançon, ${ }^{1,2}$ H. C. Walker, ${ }^{3}$ E. Ressouche, ${ }^{4}$ B. Ouladdiaf, ${ }^{1}$ K. C. Rule, ${ }^{5}$ G. J. McIntyre, ${ }^{5}$ T. J. Hicks, ${ }^{6}$ \\ H. M. Rønnow, ${ }^{2}$ and A. R. Wildes ${ }^{1, *}$ \\ ${ }^{1}$ Institut Laue-Langevin, CS 20156, 38042 Grenoble Cédex 9, France \\ ${ }^{2}$ Ecole Polytechnique Fédérale de Lausanne, SB ICMP LQM, CH-1015 Lausanne, Switzerland \\ ${ }^{3}$ ISIS facility, Rutherford Appleton Laboratory, Harwell Oxford, Didcot OX11 OQX, UK \\ ${ }^{4}$ CEA-Grenoble/INAC, Laboratoire MDN, 17 rue des martyrs, 38054 Grenoble Cédex 9, France \\ ${ }^{5}$ Australian Nuclear Science and Technology Organisation, Locked Bag 2001, Kirrawee DC NSW 2232, Australia \\ ${ }^{6}$ School of Physics, Monash University, Box 27, Clayton, Vic 3800, Australia
}

(Received 12 October 2016; published 7 December 2016)

\begin{abstract}
Neutron scattering from single crystals has been used to determine the magnetic structure and magnon dynamics of $\mathrm{FePS}_{3}$, an $S=2$ Ising-like quasi-two-dimensional antiferromagnet with a honeycomb lattice. The magnetic structure has been confirmed to have a magnetic propagation vector of $\mathbf{k}_{M}=\left[01 \frac{1}{2}\right]$ and the moments are collinear with the normal to the $a b$ planes. The magnon data could be modeled using a Heisenberg Hamiltonian with a single-ion anisotropy. Magnetic interactions up to the third in-plane nearest neighbor needed to be included for a suitable fit. The best fit parameters for the in-plane exchange interactions were $J_{1}=1.46, J_{2}=-0.04$, and $J_{3}=-0.96 \mathrm{meV}$. The single-ion anisotropy is large, $\Delta=2.66 \mathrm{meV}$, explaining the Ising-like behavior of the magnetism in the compound. The interlayer exchange is very small, $J^{\prime}=-0.0073 \mathrm{meV}$, proving that $\mathrm{FePS}_{3}$ is a very good approximation to a two-dimensional magnet.
\end{abstract}

DOI: 10.1103/PhysRevB.94.214407

\section{INTRODUCTION}

The $\mathrm{MPS}_{3}$ transition metal chalcogenophosphates $(\mathrm{M}=$ transition metal) are a family of compounds that display twodimensional characteristics in both their chemical structure and, when applicable $(\mathrm{M}=\mathrm{Mn}, \mathrm{Fe}, \mathrm{Co}, \mathrm{Ni})$, their magnetism $[1,2]$. The compounds form layered structures, and the layers are weakly bound by van der Waals forces. The members of the family all share the monoclinic space group $C \frac{2}{m}$ [3]. The transition metal ions form a planar honeycomb structure, each enclosed in an octahedron with sulfur atoms at the vertices, and a pair of phosphorus atoms are located at the center of each hexagon. The low-dimensional nature of the compounds makes them candidates for a number of applications, and they have been investigated for potential use as photorefractive materials [4] and as host lattices for intercalation [1]. More recently, efforts have been made to exfoliate individual layers with an aim to exploit their magnetic properties in graphene technology [5-9].

Their low-dimensional nature also means that the compounds are candidates for model magnetic systems. $\mathrm{FePS}_{3}$ is of particular interest, as it appears to be a very good example of a two-dimensional Ising antiferromagnet on a honeycomb lattice [10-12]. The compound forms an antiferromagnetic structure below a Néel temperature of $T_{N} \sim 120 \mathrm{~K}$, with the high-spin $(S=2) \mathrm{Fe}^{2+}$ moments pointing normal to the $a b$ planes. Neutron diffraction shows clear Bragg peaks whose temperature-dependence follows the predicted behavior for a two-dimensional Ising model [12].

The magnetic structure for $\mathrm{FePS}_{3}$ has been the subject of some controversy. Initial neutron scattering experiments, carried out on a composite sample of stacked crystals with $\mathbf{c}^{*}$ normal to the scattering plane, concluded that the

\footnotetext{
*wildes@ill.fr
}

magnetic structure had a propagation vector of $\mathbf{k}_{M}=\left[01 \frac{1}{2}\right]$ $[10,13]$. More recent neutron scattering measurements on a crystal that was known to be twinned concluded that the magnetic propagation vector was $\mathbf{k}_{M}=\left[\frac{1}{2} \frac{1}{2} 0.34\right]$ [12]. The magnetic structures in the $a b$ planes for both propagation vectors consist of ferromagnetic chains that are antiferromagnetically coupled. The two in-plane structures differ by a rotation of $120^{\circ}$ about the normal to the planes. For the interplanar structures: the $\mathbf{k}_{M}=\left[01 \frac{1}{2}\right]$ structure has a straight-forward antiferromagnetic correlation between layers, while $\mathbf{k}_{M}=\left[\frac{1}{2} \frac{1}{2} 0.34\right]$ requires an incommensurate static fluctuation of either the moment size or orientation along $\mathbf{c}$.

Neutron inelastic scattering on a powdered sample [14] showed that the magnons have a large energy gap of $\sim 16 \mathrm{meV}$ due to a large anisotropy, hence giving rise to the Ising-like behavior in the compound. The magnons are dispersive, with a band width of $16 \lesssim \Delta E \lesssim 40 \mathrm{meV}$. The magnetic density-ofstates was extracted and fitted with a two-dimensional model to give magnitudes for the anisotropy and the in-plane exchange parameters. Exchange interactions up to the third-nearest neighbor needed to be considered in order to model the data correctly. No value for the interlayer exchange could be determined by using this model.

Recent measurements on $\mathrm{NiPS}_{3}$, a sister compound of $\mathrm{FePS}_{3}$, also showed that the act of grinding these compounds into a powder may have an impact on their magnetic properties [15]. Consequently, a concerted effort was made to perform neutron scattering experiments on large single crystals in order to verify the magnetic structure and the dynamic structure factor for $\mathrm{FePS}_{3}$. We report here the result of that effort. The first part of the article describes the results of the magnetic structure determination. The second part describes the neutron inelastic scattering and the modeling of the magnon dynamics. 


\section{EXPERIMENTS}

\section{A. Sample preparation}

Single crystals of $\mathrm{FePS}_{3}$ were prepared using a vapor transport method. Quartz tubes were subjected to a cleaning procedure of etching with acid, followed by rinsing with demineralized water, followed by being heated under vacuum to $1000^{\circ} \mathrm{C}$ for $30 \mathrm{~min}$. Stoichiometric quantities of the pure elements $(\geqslant 99.998 \%)$ totaling $5 \mathrm{~g}$ were placed in the tubes, which were then evacuated and sealed before being placed in a horizontal two-zone furnace. The end of the tube containing the elements was placed in zone 1 of the furnace, and the empty end was placed in zone 2. The temperatures of the two zones were independently controlled. A heating protocol, summarized in Table I, was followed before the furnace was switched off and the tube allowed to cool.

A resulting tube contained a large number of platelet crystals with the characteristic hexagonal motif and metallic gray color of $\mathrm{FePS}_{3}$. A number of tubes were prepared resulting in the preparation of numerous large crystals with typical dimensions $\sim 10 \times 10 \times 0.2 \mathrm{~mm}^{3}$. The largest crystals were put aside for neutron diffraction and spectroscopy measurements. Some crystals were ground into a powder using a mortar and pestle for neutron powder diffraction measurements.

\section{B. Sample alignment}

A backscattering x-ray Laue diffractometer (MWL110, Multiwire Laboratories, Ltd.) at the Ecole Polytechnique Fédérale de Lausanne, Switzerland, was used to test the crystalline quality and orientation of the samples. $\mathrm{FePS}_{3}$ crystals form as platelets with the $\mathbf{c}^{*}$ axis normal to the plane, which was also the direction of the incident beam.

The Bragg peaks for $\mathrm{FePS}_{3}$ may be indexed using either a monoclinic or a hexagonal space group, each having the same c* direction. Detailed x-ray diffraction measurements were necessary to establish that the correct space group for $\mathrm{FePS}_{3}$ is the monoclinic $C \frac{2}{m}$ [3]. Laue images with the incident beam along $\mathbf{c}^{*}$, however, show a threefold symmetry and it was impossible to determine the unique $\mathbf{a}$ and $\mathbf{b}$ axes, nor whether the crystals were twinned. A monoclinic $(0 k 0)$ direction could be identified for each of the crystals, but it could equally have been a $(h h 0)$ or $(h \bar{h} 0)$ direction.

The majority of these crystals were co-aligned in a nominal $(0 k 0),(00 l)$ plane. These were used for the time-of-flight spectroscopy measurements, with the understanding that the eventual scattering would be a superposition of three twinned domains.

Some of the best and largest crystals were measured using the KOALA neutron Laue diffraction at ANSTO, Australia. This instrument has a cylindric detector and covers a solid

TABLE I. The set temperatures and durations of the two-zone growth furnace used for the synthesis of $\mathrm{FePS}_{3}$.

\begin{tabular}{lccc}
\hline \hline Stage & Zone $1\left({ }^{\circ} \mathrm{C}\right)$ & Zone $2\left({ }^{\circ} \mathrm{C}\right)$ & Duration (days) \\
\hline 1 & 700 & 750 & 12 \\
2 & 670 & 620 & 21 \\
\hline \hline
\end{tabular}

angle of $\sim 8$ steradians, giving a very large number of spots and allowing an accurate determination of the crystal quality and orientation. Two crystals were shown to be of relatively high quality. While their mosaic spreads were $\gtrsim 2^{\circ}$, analysis of the Laue diffraction showed that they were untwinned to within the accuracy of the indexing. These crystals were used to determine the magnetic structure using neutron single-crystal diffraction, and for neutron three-axis spectroscopy.

\section{Neutron diffraction}

Neutron powder diffraction with polarization analysis was carried out using the D7 spectrometer at the Institut LaueLangevin (ILL), France [16]. The neutron wavelength was set to $3.1 \AA$ using a graphite monochromator. The instrument uses supermirrors to determine the incident and final neutron spin state. Spin-flip scattering is measured with the aid of a precession-coil flipper before the sample. A liquid-helium cryostat was used to control the temperature. Measurements were performed with the neutron polarization axis normal to the scattering plane. In this configuration, the spin-flip cross-sections are free of nuclear coherent contribution and contain only magnetic and isotropic nuclear-spin-incoherent contributions. The instrument was calibrated using the scattering from amorphous quartz and vanadium, to determine the corrections for polarization inefficiencies and detector efficiency, respectively.

A preliminary study of the two nominally untwinned single crystals, co-aligned on a common mount, was performed using the IN3 three-axis spectrometer at the ILL. IN3 was configured with a pyrolytic graphite (PG) (002) monochromator and analyzer and the neutron wavelength was $2.36 \AA$. The beam divergences before and after the sample were constrained using $40^{\prime}$ collimators. Higher-order $\lambda / n$ contamination was suppressed using an oriented graphite filter. A liquid-helium cryostat was used for temperature control.

A comprehensive neutron single-crystal diffraction experiment was performed using the D10 diffractometer, also at the ILL, with a neutron wavelength of $2.36 \AA$. Higherorder contamination from the graphite monochromator was suppressed with the aid of a graphite filter. One of the nominally untwinned crystals was mounted in a liquid-helium flow cryostat on a four-circle Eulerian cradle [17]. The sample was aligned and a number of reciprocal space positions were investigated to search for the presence of twinning in the sample. These measurements were performed with a graphite analyzer between sample and detector to improve the signal-to-noise ratio. The measurements determined that the sample was indeed twinned, with three domains separated by a rotation of $120^{\circ}$ about $\mathbf{c}^{\star}$. The twinning is consistent with recent observations using $\mathrm{x}$-ray and electron diffraction [18]. The sample predominantly consisted of one domain, however, with population ratios between the domains of $0.78: 0.13: 0.09$. The sample alignment was then adapted to focus on the majority domain. A position-sensitive detector with no energy analysis was used to collect the Bragg peak intensities for a number of crystal and magnetic reflections at $2 \mathrm{~K}$. The data were reduced using the RACER software [19], and the crystal and magnetic structures were determined using the FullProf software suite [20]. 


\section{Three-axis spectrometry}

Neutron three-axis spectrometry was used to determine the magnon dispersion along the $\mathbf{c}^{\star}$ direction. The two crystals set aside from neutron Laue diffraction were wrapped in aluminium foil envelopes before being glued to aluminium pins. The crystals were then co-aligned before the pins were fixed to a common sample mount.

Preliminary measurements were performed using the TAIPAN spectrometer at ANSTO, Australia [21], configured with a PG (002) monochromator and analyzer and using 40' collimators before and after the sample. An oriented graphite filter was used to suppress $\lambda / n$. Temperature control was achieved using cryorefrigerator.

The magnon dispersion parallel to $\mathbf{c}^{*}$ was measured using the IN8 [22] and IN20 [23] spectrometers at the Institut Laue-Langevin, France. Measurements for energy transfers up to $\Delta(E) \leqslant 28 \mathrm{meV}$ were performed on each instrument using a bent silicon (111) monochromator and a focused PG (002) analyser. IN8 was fitted with a focused copper (200) monochromator to measure energy transfers between $30 \leqslant$ $\Delta E \leqslant 50 \mathrm{meV}$. Both instruments used an oriented graphite filter between sample and analyzer for $\lambda / n$ suppression. A liquid-helium cryostat was used for temperature control.

\section{E. Time-of-flight spectroscopy}

Time-of-flight neutron spectroscopy was used to measure the magnon spectrum in the plane normal to the $\mathbf{c}^{*}$ axis. Neutron scattering from a composite crystal was measured using the MERLIN direct-geometry neutron chopper spectrometer at the ISIS facility, UK [24]. Numerous large-platelet crystals were glued to thin aluminium sheets with small amounts of CYTOP fluoropolymer glue [25]. The $\mathbf{c}^{\star}$ axis is normal to the platelets and was therefore normal to the aluminium sheets. The samples were nominally mounted to have the $(0 k 0)$ direction in the horizontal plane, however, as x-ray Laue diffraction was not able to define this direction unambiguously and the crystals were most probably twinned, the horizontal plane also contained the $(h h 0)$ or $(h \bar{h} 0)$ directions. The scattering was therefore expected to show a superposition of the magnetic contributions from three twinned domains.

The aluminium sheets were then mounted on a "toaster rack" aluminium block. The block held 9 sheets with a total of 17 crystals amounting to an estimated mass of $0.3 \mathrm{~g}$. The sample temperature was controlled down to $5 \mathrm{~K}$ using a cryorefrigerator.

The sample was mounted on the instrument to have the $\mathbf{c}^{\star}$ axis parallel to the incident wave vector $\mathbf{k}_{i}$. If the magnons along $\mathbf{c}^{\star}$ were weakly or not dispersive, as was expected for $\mathrm{FePS}_{3}$, then the scattering along this direction could be integrated and the data plotted as a function of energy and those components of $\mathbf{Q}$ in the $a b$ planes. Similar geometries have been adopted for many other quasi-two-dimensional magnetic materials [26-28].

The instrument was configured to run in repetition-rate multiplication mode $[29,30]$. In this mode the source pulse and incident beam chopper are synchronized such that one source pulse provides neutrons with up to three discrete energies at the sample, $E_{i}$, each arriving at a separate time. The time that the neutrons arrive at the detector is measured and correlated to its respective $E_{i}$, as well as determining the final energy, $E_{f}$. The mode permits spectra with different resolutions and kinematic constraints to be simultaneously measured. Two incident energies were simultaneously measured in the experiment: $E_{i}=31.8 \mathrm{meV}$, which gave a higher resolution measurement around the spin wave gap energy at $\Delta E \sim 15 \mathrm{meV}$; and $E_{i}=75 \mathrm{meV}$, which gave an overview of the entire magnon spectrum [14].

\section{RESULTS}

\section{A. The magnetic structure}

The preliminary neutron elastic scattering data at $2 \mathrm{~K}$, measured using the IN3 spectrometer, are shown in Fig. 1. The measurements were performed on both of the nominally untwinned crystals that had been co-aligned on a common sample mount. The figure shows scans along $\left(\frac{1}{2} \frac{1}{2} l\right)$, $(01 l)$, and $\left(\frac{1}{2} \frac{1}{2} l\right)$. All the measurements show sharp Bragg peaks at noninteger positions. The peaks in the scans along $\left(\frac{1}{2} \frac{1}{2} l\right)$ and $\left(\frac{1}{2} \frac{1}{2} l\right)$ are at $\left(N+\frac{1}{3}\right)$, where $N$ is an integer. No peaks appear at $\left(N-\frac{1}{3}\right)$ in these scans. The peaks along $(01 l)$ are more than an order of magnitude larger in intensity and are at $\left(N \pm \frac{1}{2}\right)$.

The data establish that the magnetic propagation vector for $\mathrm{FePS}_{3}$ cannot be $\mathbf{k}_{M}=\left[\frac{1}{2} \frac{1}{2} 0.34\right]$. This propagation vector would give rise to Bragg peaks at $\sim\left(N-\frac{1}{3}\right)$ in the scans along $\left(\frac{1}{2} \frac{1}{2} l\right)$. Instead, the correct magnetic propagation vector is $\mathbf{k}_{M}=\left[01 \frac{1}{2}\right]$, which gives the observed magnetic Bragg peaks along $(01 l)$. The observed Bragg peaks along $\left(\frac{1}{2} \frac{1}{2} l\right)$ and $\left(\frac{1}{2} \frac{1}{2} l\right)$

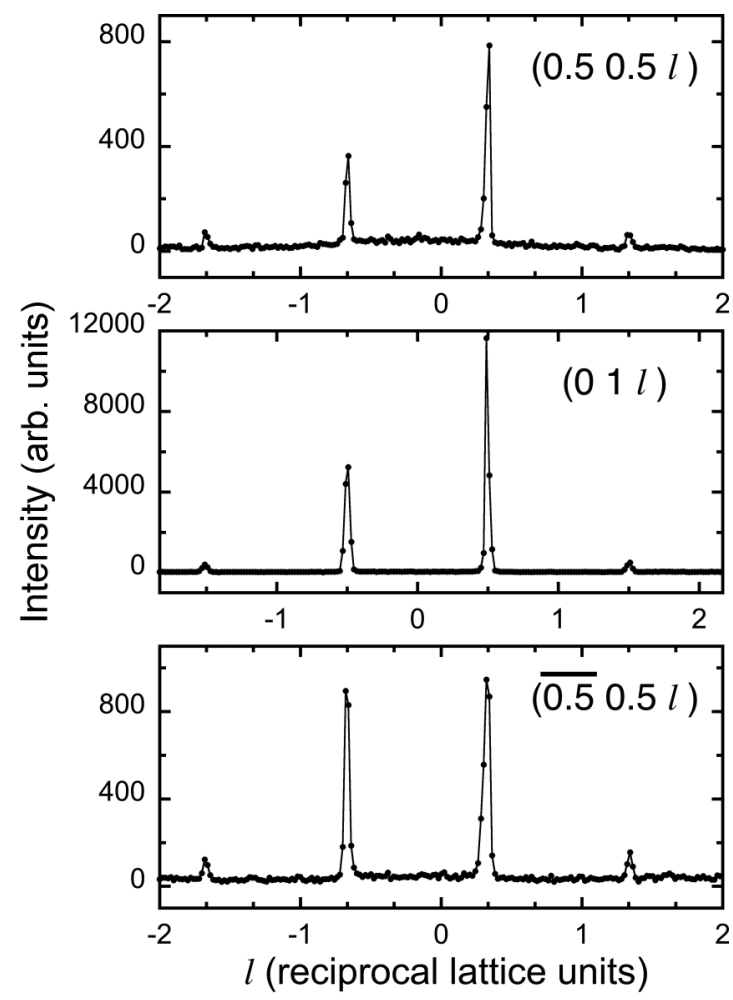

FIG. 1. Neutron elastic scattering scans along $l$ for three fixed $(h k)$, measured using IN3. The successive $(h k)$ correspond to $60^{\circ}$ rotations about $\mathbf{c}^{*}$. All the data were measured at $2 \mathrm{~K}$. 

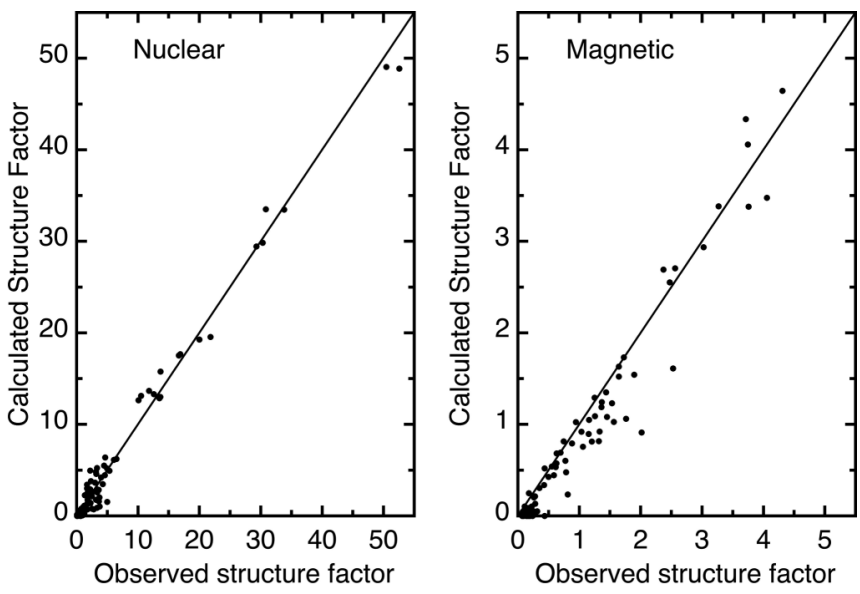

FIG. 2. The observed versus calculated crystal and magnetic structure factors for $\mathrm{FePS}_{3}$ at $2 \mathrm{~K}$ from the refinement of neutron scattering data from D10. The crystal structure refinement was performed first, and the resulting atomic coordinates and thermal factors were fixed in the refinement of the magnetic data.

are due to the $120^{\circ}$ crystal twins. If they are indexed using the appropriate twin, they map onto the $\mathbf{k}_{M}=\left[01 \frac{1}{2}\right]$ propagation vector. The Appendix describes the rotation matrices for indexing these peaks in the presence of twinned domains.

While IN3 is suboptimal for the determination of Bragg peak intensities, the magnetic Bragg peaks along (01l) are at least an order of magnitude larger than those along the other two axes. This confirms that the crystals predominantly consisted of a single domain, and the ratios of the magnetic intensities roughly correspond to the twin domain population ratios determined for one of the two crystals using D10. The Bragg peaks in Fig. 1 are much sharper in $l$ than those shown in previous data [12], indicating that the magnetic order is long-ranged. The difference can be attributed to the improved quality of the crystals.

The magnetic structure was confirmed from the measurements of one of the crystals on D10. The crystal structure was refined from data collected at $2 \mathrm{~K}$, starting from the parameters given by Ouvrard et al. [3]. The size and quality of the crystal limited the accuracy of the refinement; however, the final structural parameters were consistent with those in the literature. The results are summarized in Fig. 2 and the best parameters are listed in Table II.

TABLE II. The refined crystal structure parameters for $\mathrm{FePS}_{3}$ at $2 \mathrm{~K}$. The space group was $C 2 / m$, with lattice parameters $a=$ 5.94(4) $\AA$, $b=10.26(2) \AA, c=6.60(6) \AA$, and $\beta=108.3(7)^{\circ}$. The fractional coordinates are given with respect to the monoclinic unit cell. The residuals were ${ }_{w} R_{f 2}=10.7 \%$ and $\chi^{2}=5.29$.

\begin{tabular}{lccccc}
\hline \hline \multirow{2}{*}{ Atom } & $\begin{array}{r}\text { Wyckoff } \\
\text { position }\end{array}$ & $x$ & $y$ & $z$ & $B_{\text {iso }}$ \\
\hline $\mathrm{Fe}$ & $4 g$ & 0 & $0.327(3)$ & 0 & $0.665(2)$ \\
$\mathrm{P}$ & $4 i$ & $0.051(5)$ & 0 & $0.164(2)$ & $0.205(2)$ \\
$\mathrm{S}(1)$ & $4 i$ & $0.720(1)$ & 0 & $0.192(5)$ & $0.455(2)$ \\
$\mathrm{S}(1)$ & $8 j$ & $0.257(6)$ & $0.165(6)$ & $0.267(3)$ & $0.455(2)$ \\
\hline \hline
\end{tabular}

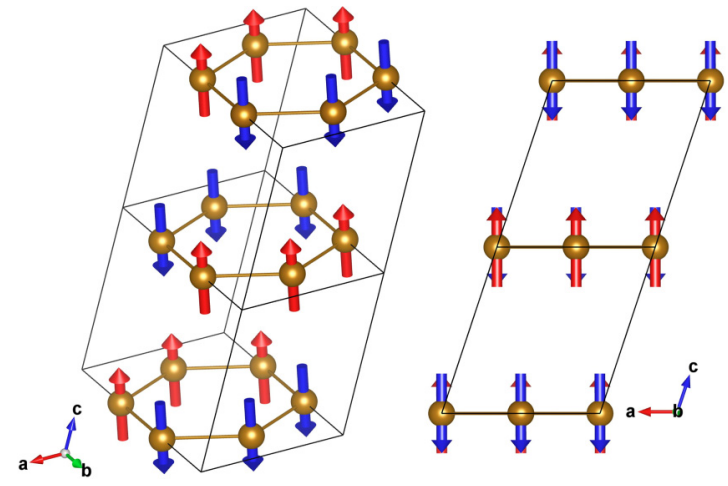

FIG. 3. The magnetic structure for $\mathrm{FePS}_{3}$, shown for two orientations. The moments all have a magnitude of $4.52 \pm 0.05 \mu_{B}$ and are color-coded to differentiate between the two moment orientations. The figure was created using the VESTA program [31].

The BasIreps program within the FullProf suite was used to determine the irreducible representations compatible with the crystal symmetry and a propagation vector of $\left[01 \frac{1}{2}\right]$. The moment components and symmetry operators for the resulting four representations are shown in Table III. The magnetic structure was refined using the structural and thermal parameters from the crystal structure refinement. As is evident from the residuals listed in the Table III, Irep(4) gives by far the best match to the magnetic Bragg peak intensities. The results of the refinement with Irep(4) are shown in Fig. 2. The refined moment direction was normal to the $a b$ planes within an accuracy of $\pm 2^{\circ}$, as expected from previous studies [10,12]. The moment magnitude was $4.52 \pm 0.05 \mu_{B}$, also consistent with previous studies and with the expected moment for $S=2$ high-spin-state $\mathrm{Fe}^{2+}$. A schematic of the magnetic structure for $\mathrm{FePS}_{3}$ is shown in Fig. 3.

\section{B. The magnon dynamics}

\section{The magnetic dynamic structure factor}

A magnetic dynamic structure factor, $S_{\mathrm{mag}}(\mathbf{Q}, \omega)$, has previously been calculated for $\mathrm{FePS}_{3}$ and was used to determine the magnetic exchange parameters using neutron inelastic scattering data from a powdered sample [14]. The structure factor was derived from a Heisenberg Hamiltonian with a (large) single-ion easy-axis anisotropy:

$$
H=-\sum_{i, j} J_{i, j} \mathbf{S}_{i} \cdot \mathbf{S}_{j}-\Delta \sum_{i}\left(S_{i}^{z}\right)^{2} .
$$

The calculation assumed that the magnetic structure was purely two-dimensional. Exchange between the $a b$ layer planes was neglected.

A full calculation of $S_{\mathrm{mag}}(\mathbf{Q}, \omega)$ requires the inclusion of an interlayer exchange. Hence, the equations previously published [14] must be expanded to three dimensions.

The calculation may be performed by decomposing the magnetic structure into interlocking primitive lattices. Four such lattices were required to describe the magnetic structure in two dimensions [14]. Four lattices can equally be used to describe the magnetic structure in three dimensions. Figure 4 
TABLE III. The moment components and symmetry operators associated with the four possible irreducible representations for a magnetic propagation vector of $\left[01 \frac{1}{2}\right]$, along with the residuals from fitting the D10 data with each representation. Note that C-centering is respected.

\begin{tabular}{|c|c|c|c|c|c|}
\hline & & $\operatorname{IRep}(1)$ & $\operatorname{IRep}(2)$ & $\operatorname{IRep}(3)$ & $\operatorname{IRep}(4)$ \\
\hline \multicolumn{6}{|l|}{ Moments: } \\
\hline & $(x, y, z)$ & $\left(0, M_{y}, 0\right)$ & $\left(0, M_{y}, 0\right)$ & $\left(M_{x}, 0, M_{z}\right)$ & $\left(M_{x}, 0, M_{z}\right)$ \\
\hline & $(\bar{x}, 1+\bar{y}, \bar{z})$ & $\left(0, M_{y}, 0\right)$ & $\left(0,-M_{y}, 0\right)$ & $\left(M_{x}, 0, M_{z}\right)$ & $\left(-M_{x}, 0,-M_{z}\right)$ \\
\hline \multicolumn{6}{|c|}{ Symmetries: } \\
\hline & $1:(0,0,0)$ & 1 & 1 & 1 & 1 \\
\hline & $2:(0, y, 0)$ & 1 & 1 & -1 & -1 \\
\hline & $-1:(0,0,0)$ & 1 & -1 & 1 & -1 \\
\hline & $\mathrm{m}:(x, 0, z)$ & 1 & -1 & -1 & 1 \\
\hline \multicolumn{6}{|l|}{ Residuals: } \\
\hline & ${ }_{w} R_{f 2}$ & 98.9 & 59.7 & 95.4 & 21.0 \\
\hline & $\chi^{2}$ & 548.4 & 199.8 & 539.5 & 24.9 \\
\hline
\end{tabular}

shows the monoclinic unit cell for $\mathrm{FePS}_{3}$, along with one of the interlocking primitive lattices for the magnetic structure.

With the exception of a, the primitive lattices do not have the same lattice vectors as the monoclinic unit cell. The Miller indices for the two lattices are related through the transformation:

$$
\left[\begin{array}{l}
h \\
k \\
l
\end{array}\right]=\left[\begin{array}{lll}
1 & 0 & 0 \\
1 & 1 & 0 \\
0 & \frac{1}{2} & 1
\end{array}\right]\left[\begin{array}{l}
h_{\mathrm{mag}} \\
k_{\mathrm{mag}} \\
l_{\mathrm{mag}}
\end{array}\right]
$$

where the subscript mag denotes the magnetic primitive lattice.

The exchange parameters are defined according to the nearest-neighbor distances. They are shown schematically in Fig. 5. The exchange parameters up to the third-nearest neighbor in the $a b$ planes were required to describe the neutron inelastic scattering from powdered $\mathrm{FePS}_{3}$ [14]. The same parameters, labeled $J_{1}, J_{2}$, and $J_{3}$ for first-, second-, and third-nearest neighbors, respectively, were used here. Each $\mathrm{Fe}^{2+}$ ion has four nearest neighbors between the $a b$ planes. They are split into two pairs, each pair belonging to a different primitive sublattice. The possible exchange pathways through the intervening sulfur layers are almost identical, and hence only one interplanar exchange parameter, $J^{\prime}$, was used.

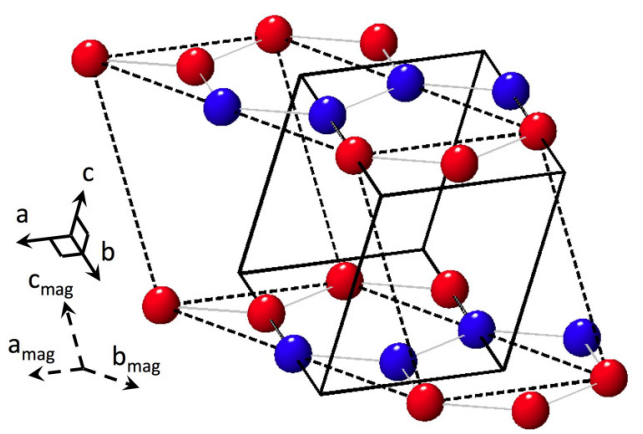

FIG. 4. The monoclinic unit cell (solid lines), and one of the four interlocking primitive lattices (dashed lines) used to construct the magnetic structure for the calculation of $S(\mathbf{Q}, \omega)$. The red spheres indicate those $\mathrm{Fe}^{2+}$ ions with moments up, and the blue indicate those with moments down. The lattice parameters for the magnetic unit cell are approximately $a_{\mathrm{mag}}=a, b_{\mathrm{mag}}=2 a, c_{\mathrm{mag}}=\frac{a}{3} \sqrt{7+\tan ^{2} \beta}$, $\alpha_{\mathrm{mag}}=126.7^{\circ}, \beta_{\mathrm{mag}}=83.1^{\circ}, \gamma_{\mathrm{mag}}=120^{\circ}$, with $\mathbf{a}_{\mathrm{mag}}=\mathbf{a}$.
After applying the Holstein-Primakoff transformation, expanding and Fourier transforming, the Hamiltonian may be written in an identical form as in Ref. [14] with modifications of the matrix elements to account for the interplanar exchange:

$$
\begin{aligned}
\mathbf{H}_{M}= & 2 S\left[\begin{array}{cccc}
A & B^{*} & C & D^{*} \\
B & A & D & C \\
C & D^{*} & A & B^{*} \\
D & C & B & A
\end{array}\right], \\
A= & 2 J_{2} \cos \left(2 \pi h_{\mathrm{mag}}\right) \\
& -\Delta-J_{1}+2 J_{2}+3 J_{3}+4 J^{\prime} \\
B= & J_{1} \exp \left[\frac{2 \pi i}{3}\left(2 h_{\mathrm{mag}}+\frac{k_{\mathrm{mag}}}{2}\right)\right] \\
& \times\left[1+\exp \left(-2 \pi i h_{\mathrm{mag}}\right)\right], \\
C= & 2 J_{2}\left\{\cos \left(\pi k_{\mathrm{mag}}\right)+\cos \left[2 \pi\left(h_{\mathrm{mag}}+\frac{k_{\mathrm{mag}}}{2}\right)\right]\right\} \\
& +J^{\prime} \cos \left[\pi\left(k_{\mathrm{mag}}+2 l_{\mathrm{mag}}\right)\right],
\end{aligned}
$$

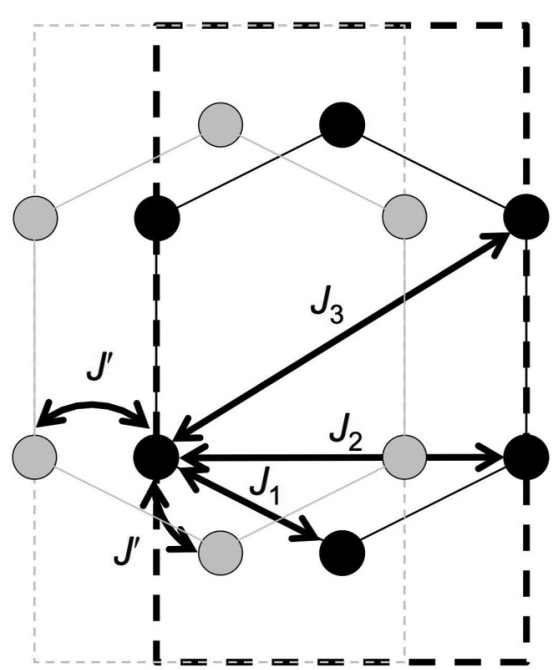

FIG. 5. View down the $\mathbf{c}^{\star}$ axis for $\mathrm{FePS}_{3}$, showing the inter- and intraplanar exchange parameters. The black circles represent the $\mathrm{Fe}^{2+}$ positions in the $a b$ basal plane, while the gray circles are the $\mathrm{Fe}^{2+}$ positions in the plane displaced one lattice unit along $\mathbf{c}$. 

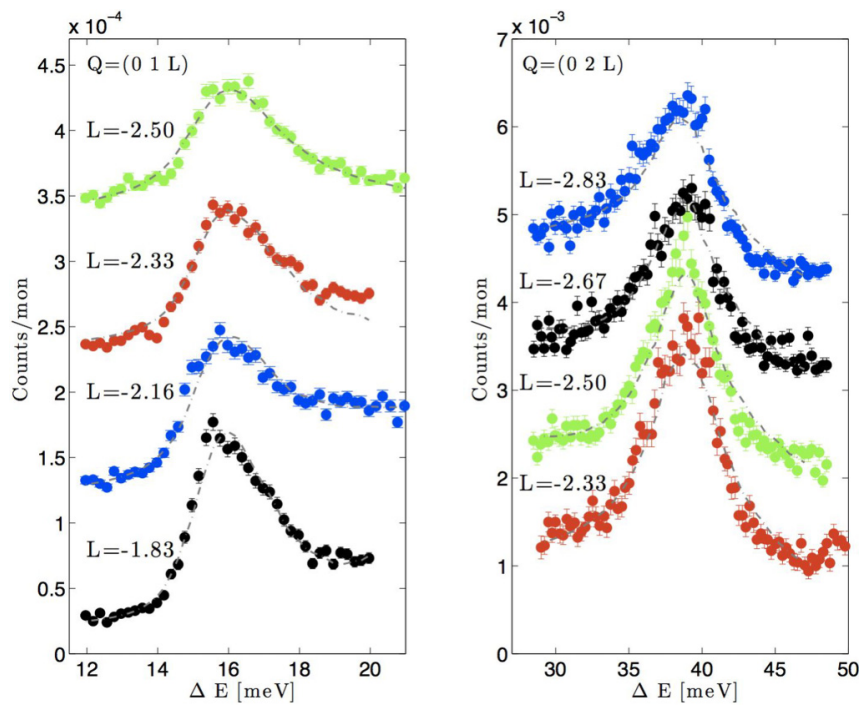

FIG. 6. Constant-Q scans at (01l) using IN20 (left) and (02l) using IN8 (right) for various values of $l$. Fits to the data of $S_{\operatorname{mag}}(\mathbf{Q}, \omega)$ convoluted with the instrument resolution are shown as dashed lines. The spectra are shifted vertically for clarity.

$$
\begin{aligned}
& D=\exp \left[\frac{2 \pi i}{3}\left(2 h_{\mathrm{mag}}+\frac{k_{\mathrm{mag}}}{2}\right)\right]
\end{aligned}
$$

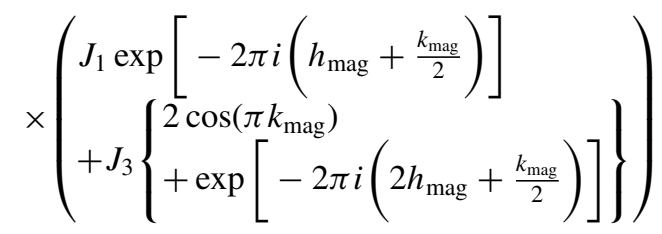

$$
\begin{aligned}
& +2 J^{\prime} \exp \left[\frac{\pi i}{3}\left(h_{\mathrm{mag}}+k_{\mathrm{mag}}\right)\right] \\
& \times \cos \left[\pi\left(h_{\text {mag }}+k_{\text {mag }}+2 l_{\text {mag }}\right)\right] .
\end{aligned}
$$

The values for $A$ and $B$ are identical to those in Ref. [14]. Interlayer exchange requires modification of $C$ and $D$. Subject to these modifications, the eigenvalues and eigenvectors for

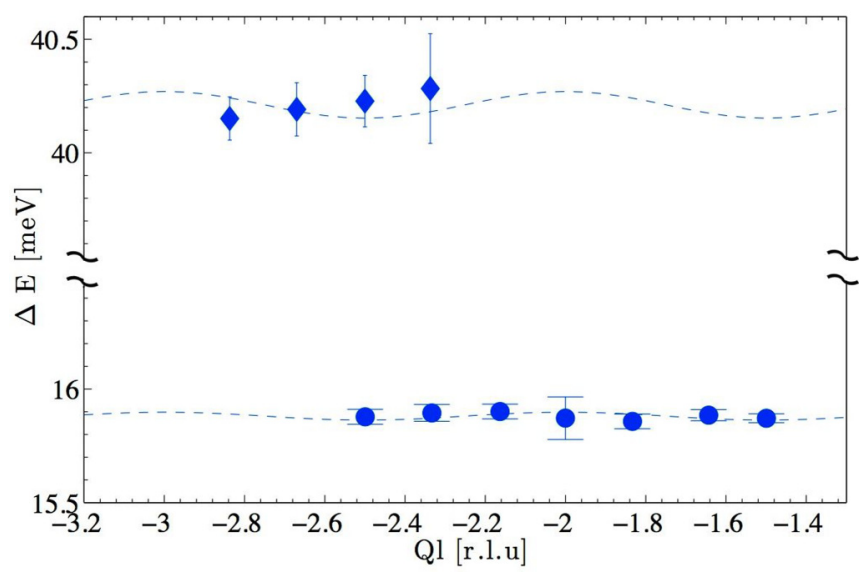

FIG. 7. The magnon energies as a function of $l$. The data at $\Delta E \sim$ $15.8 \mathrm{meV}$ result from fitting the magnons along $(01 \mathrm{l})$. Note that the magnetic Bragg peaks are found at $\left(01 \frac{\overline{3}}{2}\right)$ and $\left(01 \frac{\overline{5}}{2}\right)$. The data at $\Delta E \sim 40.3 \mathrm{meV}$ result from the magnons along $(02 l)$.
Eq. (3) are identical to those in Ref. [32]. We also note that the form of Eq. (3) is identical to that described by Wheeler et al. [33]. These authors have included explicit equations for the eigenvectors that are equally applicable to $\mathrm{FePS}_{3}$.

\section{Three-axis spectrometry and the magnon dispersion along $\mathrm{c}^{*}$}

Constant-Q measurements were performed at various points along $(01 l)$, through the Brillouin zone center, and along $(02 l)$, at a Brillouin zone boundary. A selection of the data are shown in Fig. 6. The data show strong peaks due to magnons, which were fitted using a function consisting of a damped harmonic oscillator convoluted with the instrument resolution. The fits are also shown in the Fig. 6. The width of the damped harmonic oscillator was a fit parameter, although the fitted value depended on the mosaic spread for the crystal used in the resolution calculation. The combined mosaic spread for the two coaligned crystals was roughly $2^{\circ}$, although the peak shape was quite irregular, and the mean fitted width with this value was $0.58 \mathrm{meV}$. This width was subsequently fixed and the data refitted to find the characteristic energies, which are plotted as a function of $l$ in Fig. 7.

The magnons have a weak dispersion along the $\mathbf{c}^{*}$ direction. The data were used to extract the interlayer exchange parameter by fitting the eigenvalues derived from Eqs. (3) and the best value was found to be $J^{\prime}=-0.0073 \pm 0.0003 \mathrm{meV}$. The interlayer exchange is very small but is nonzero and negative. This confirms the two-dimensional characteristics of $\mathrm{FePS}_{3}$ and supports the conclusion that the layers are antiferromagnetically coupled.

\section{Time-of-flight spectrometry and the magnon dispersion in the ab planes}

The very weak spin-wave dispersion along the $\mathbf{c}^{\star}$ direction proves that $\mathrm{FePS}_{3}$ is a good approximation of a two-dimensional magnet. It was therefore valid to integrate the scattering parallel to the $\mathbf{c}^{\star}$ direction, inherent in the MERLIN experiment, to have a measure of the magnon dispersion in the $a b$ planes. The data were reduced with appropriate software [34,35] to convert units from time-of-flight and scattering angle into $S_{\text {mag }}(\mathbf{Q}, \omega)$. As expected, the data showed the superposition of the magnetic scattering from three twinned domains, rotated by $120^{\circ}$ relative to one another. The ratio of the domain populations was found to be $0.465: 0.206$ : 0.329 , estimated by comparing the integrated intensities within $15.4 \leqslant \Delta E \leqslant 15.8 \mathrm{meV}$ around the $(010)$ position for each of the three domains. The reciprocal lattice vectors were calculated and the data plotted and fitted relative to the domain with the strongest scattering. Plotting the data relative to the weaker domains gave the same results.

Representative data at $5 \mathrm{~K}$ for various slices through reciprocal space are shown in Figs. 8(a)-8(d). Figures 8(a)8 (c) show slices along $\left(h k \frac{\bar{h}}{3}\right)$, which are perpendicular to $(0 k 0)$. These data were measured using $E_{i}=75 \mathrm{meV}$. Analysis of the previous powder measurements suggested that the spin waves along this direction were highly dispersive [14], and the data show this to be the case. The magnon energies in these slices were expected to resemble two oscillating functions, each representing a pair of degenerate magnon modes, shifted by half a period relative to one another. This is indeed what 

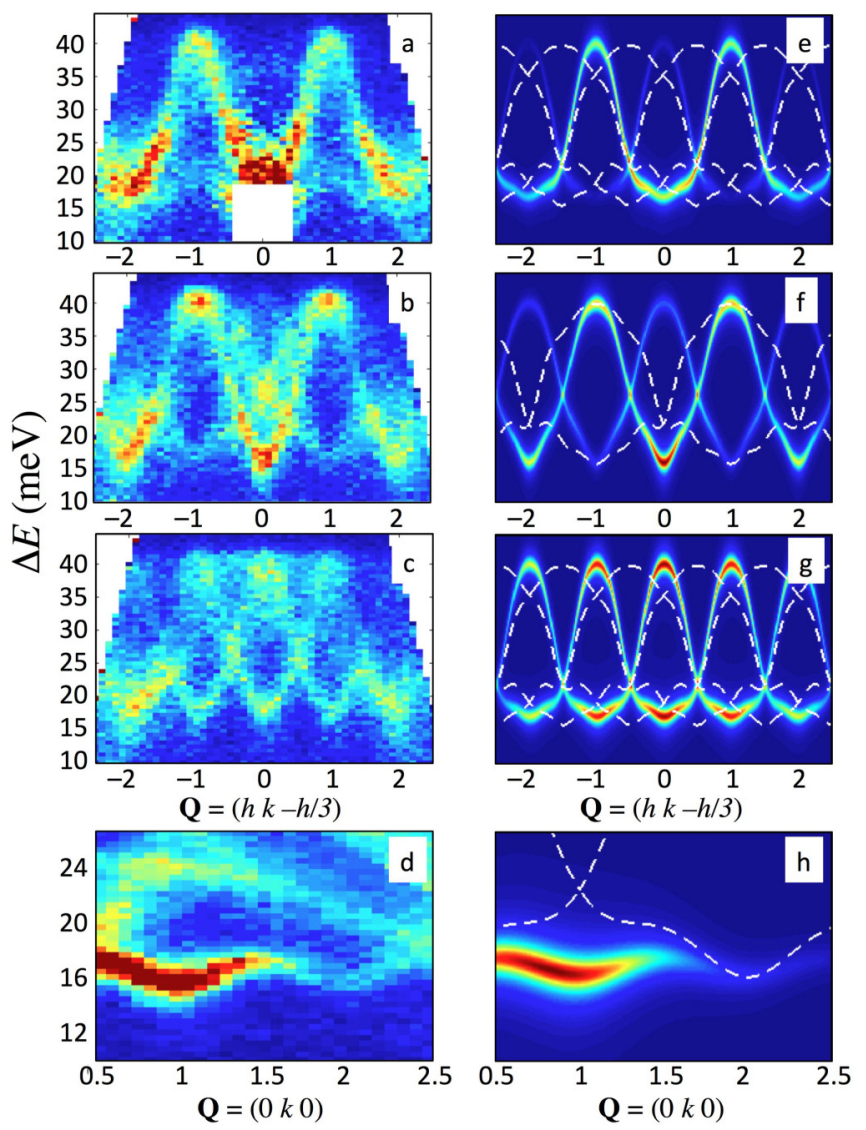

FIG. 8. Neutron inelastic data from $\mathrm{FePS}_{3}$, measured using timeof-flight spectroscopy on MERLIN at $5 \mathrm{~K}$, for various $(\mathbf{Q}, \Delta E)$ slices through reciprocal space. Panels (a)-(c) show the experimental data for slices perpendicular to the nominal $\mathbf{b}^{*}$ axis, centered at $k=\frac{1}{2}$, 1 , and $\frac{3}{2}$ respectively. Panel (d) shows data for a slice along the nominal $\mathbf{b}^{*}$ axis. Panels (e)-(h) show corresponding calculations for $S_{\text {mag }}(\mathbf{Q}, \omega)$. The magnon energies for the two $120^{\circ}$ twinned domains are shown as dashed white lines.

was observed and is most clearly visible in Fig. 8(c). The eigenvectors for one of the two oscillating functions dominate the scattering in Figs. 8(a) and 8(b) due to the magnitudes of $S_{\text {mag }}(\mathbf{Q}, \omega)$ at these positions.

Scattering from the other two twin domains is also visible in the data. Extra intensity is apparent around the minimum accessible $(\mathbf{Q}, \omega)$ in Fig. 8(a). The intensity consists of two lobes that are just visible at $\Delta E \sim 16 \mathrm{meV}$ and $\mathbf{Q} \sim\left( \pm \frac{1}{2} \frac{1}{2} \mp \frac{1}{6}\right)$, and some extra spectral weight at $\Delta E \sim 20 \mathrm{meV}$ and $\mathbf{Q} \sim\left(0 \frac{1}{2} 0\right)$. The superimposed magnons from the other domains are even more apparent in Fig. 8(b), with clearly dispersive modes that cross to give reasonably strong scattering at $\Delta E \sim 26 \mathrm{meV}$ and $\mathbf{Q} \sim(010)$.

Figure 8(d) shows a slice along ( $0 k 0)$, focusing on the spinwave gap and measured using $E_{i}=31.8 \mathrm{meV}$. The magnons give strong scattering between $15 \lesssim \Delta E \lesssim 17 \mathrm{meV}$. The spin waves in this direction were expected to have an extremely weak dispersion [14]. The dispersion is indeed weak, although it is greater than was expected from the analysis of the powder data. The scattering from the other two domains appears as a line of intensity with an apparent dispersion, increasing from
TABLE IV. Table showing the best estimates for the exchange parameters and anisotropy in $\mathrm{FePS}_{3}$ from measurements on powdered [14] and single-crystal (current work) samples. All units are in meV.

\begin{tabular}{lcc}
\hline \hline & Values from Ref. [14] & Values from current work \\
\hline$J_{1}$ & $1.49(3)$ & $1.46(1)$ \\
$J_{2}$ & $0.04(3)$ & $-0.04(4)$ \\
$J_{3}$ & $-0.6(2)$ & $-0.96(5)$ \\
$J^{\prime}$ & NA & $-0.0073(3)$ \\
$\Delta$ & $3.7(3)$ & $2.66(8)$ \\
\hline \hline
\end{tabular}

$\sim 16$ to $\sim 25 \mathrm{meV}$ between $0.5<k \leqslant 1$ and then decreasing back to $\sim 16 \mathrm{meV}$ from $1<k \leqslant 2$.

Figure $8(d)$ also shows some extra spurious scattering. An arc of intensity is visible between $\sim 24 \mathrm{meV}$ at $\left(0 \frac{1}{2} 0\right)$ and $\sim 17 \mathrm{meV}$ at $\left(0 \frac{5}{2} 0\right)$, and some extra intensity is apparent in the top right-hand corner. Measurements of an empty sample holder also showed this scattering, hence it is due to instrumental background.

One-dimensional cuts through reciprocal space were extracted to determine the magnon energies as a function of $\mathbf{Q}$. The localized nature of the contributions from other domains and spurions meant that they could easily be avoided or accounted for. The data were fitted with damped harmonic oscillator functions, and the resulting characteristic magnon energies were then fitted with the expression for the spin-wave dispersion derived from Eq. (3). The interlayer exchange, $J^{\prime}$, was included in the analysis but was fixed to the value determined from the three-axis spectrometry experiments. The best parameters are listed in Table IV, along with the previous estimates determined from neutron inelastic scattering on a powdered sample [14].

The exchange parameters $J_{1}$ and $J_{2}$ are the same, within errors, in the corresponding estimates from powdered and single crystal samples. The magnitude of the single-crystal estimate for the third-nearest-neighbor exchange, $J_{3}$, is substantially larger than that from the powder. This is a result of the dispersion of the magnon gap, evident in Fig. 8(d). The magnitude of the anisotropy correspondingly decreased when fitting the single-crystal data.

The values in Table IV were used to calculate $S_{\mathrm{mag}}(\mathbf{Q}, \omega)$, and Figs. 8(e)-8(h) show calculations for the corresponding slices through reciprocal space shown in Figs. 8(a)-8(d). The magnon energies for the other two twinned domains were also calculated and are shown as white dashed lines in the figures.

The comparison between the calculation and the data is reasonable but not perfect. The calculations are in satisfactory agreement with the data along $(0 k 0)$ and $\left(h 1 \frac{\bar{h}}{3}\right)$, shown in Figs. 8(b), 8(f) and 8(d), 8(h), respectively. However, the calculated magnon dispersion at the lowest energies in the $\left(h \frac{1}{2} \frac{\bar{h}}{3}\right)$ and $\left(h \frac{3}{2} \frac{h}{3}\right)$ slices are more rounded than the data. This causes the two oscillating magnon branches to intersect at lower energy transfer than observed. Furthermore, the magnons from the $120^{\circ}$ domains in the simulations have a weaker dispersion than the data in the low energy part of the spectra. This is a direct result of increasing $J_{3}$. Calculations using the values estimated from powdered samples give images 
that are qualitatively much better for the slices along $\left(h k \frac{\bar{h}}{3}\right)$, but there is a drastic effect on the slice along $(0 k 0)$ with the magnons becoming almost dispersionless. The values listed in Table IV represent the best compromise to have a reasonable fit along all directions in reciprocal space.

\section{DISCUSSION}

Establishing the correct magnetic structure in $\mathrm{FePS}_{3}$ is complicated by the propensity of the compound for crystal twinning. Rule et al. [12] measured a number of magnetic Bragg peaks from a twinned crystal and indexed them using the propagation vector $\mathbf{k}_{M}=\left[\frac{1}{2} \frac{1}{2} 0.34\right]$. The Bragg peaks measured in this study were rather broad along the $\mathbf{c}^{\star}$ direction. However, the peaks shown in Fig. 1 are substantially sharper. It is reasonable to suggest that the broad width along $l$ for the previous study was due to the crystal quality rather than an intrinsic feature of the magnetic structure. The propagation vector $\mathbf{k}_{M}=\left[\frac{1}{2} \frac{1}{2} 0.34\right]$ is incommensurate along $l$. Any incommensurability must be due either to components of the moments that were not collinear with the normal to the $a b$ planes, which would be surprising for such a good example of an Ising-like system, or to some periodic variation of the moment size, which would be surprising for an insulating compound consisting of high-spin $\mathrm{Fe}^{2+}$ ions. The value of $l=0.34$ might also be an artifact of the crystal quality. However, the symmetry arguments against this propagation vector equally apply if it is rationalized to $\mathbf{k}_{M}=\left[\frac{1}{2} \frac{1}{2} \frac{1}{3}\right]$.

As shown in the Appendix, magnetic peaks indexed with this propagation vector can equally be indexed using the coordinates for a twinned domain with a propagation vector of $\mathbf{k}_{M}=\left[01 \frac{1}{2}\right]$. The new propagation vector is also consistent with an Ising structure with localized moments, and with the neutron measurements of the higher-quality crystal shown in Fig. 1. It therefore appears that the correct propagation vector for $\mathrm{FePS}_{3}$ is $\mathbf{k}_{M}=\left[01 \frac{1}{2}\right]$.

There is a final discrepancy to be resolved concerning the magnetic structure. Rule et al. [36] have shown neutron powder diffraction measurements with magnetic peaks that cannot be indexed with a propagation vector of $\mathbf{k}_{M}=\left[01 \frac{1}{2}\right]$. Figure 9 shows the neutron spin-flip scattering from powdered $\mathrm{FePS}_{3}$ at $2 \mathrm{~K}$, measured using D7 with the neutron polarization normal to the scattering plane. This scattering contains two-thirds of the nuclear spin-incoherent cross-section, which give an isotropic and flat background, and part of the magnetic cross-section, which show Bragg peaks due to the magnetic structure. The magnetic peaks are mostly quite broad and show intensity tails at larger $Q$, which is characteristic of quasitwo-dimensional systems and is also observed in powdered $\mathrm{MnPS}_{3}$ [37]. The Bragg peaks can mostly be indexed with a propagation vector of $\mathbf{k}_{M}=\left[01 \frac{1}{2}\right]$, and examples are shown in Fig. 9.

However, the first peak shows substantial intensity at momentum transfers well below $\left(01 \frac{1}{2}\right)$, which is the first allowed Bragg peak for $\mathbf{k}_{M}=\left[01 \frac{1}{2}\right]$. The peak begins at a $Q$ that may be indexed with (010). Given the two-dimensional nature of the line shapes in the powder diffraction, it appears that the act of grinding the sample has induced enough stacking

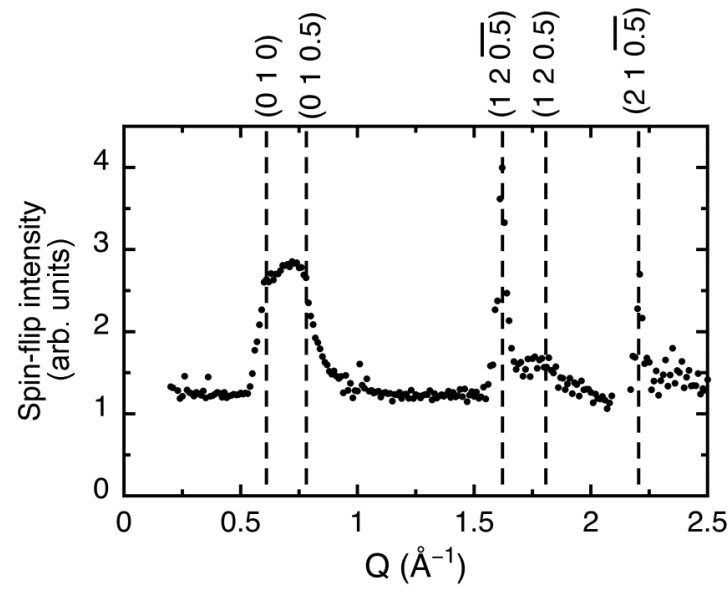

FIG. 9. Neutron spin-flip scattering from powdered $\mathrm{FePS}_{3}$ at $2 \mathrm{~K}$ using D7. The neutron polarization was normal to the scattering plane. The scattering is due to a combination of two-thirds of the nuclear spin-incoherent cross-section and, in the absence of preferred orientation, one half of the magnetic cross-section.

faults and dislocations so as to destroy any long-ranged order along the $\mathbf{c}^{\star}$ axis with the in-plane structure remaining the same. The Bragg peaks become Bragg rods, with the first rod for a $\mathrm{FePS}_{3}$ sheet appearing at (010).

The conjecture is supported by a calculation, shown in Fig. 5 of Rule et al. [12], for the magnetic scattering from a powdered sample with elongated extension along $l$. The calculation qualitatively agrees reasonably well with both their data and the data in Fig. 9. The calculation was performed for a structure with $\mathbf{k}_{M}=\left[\frac{1}{2} \frac{1}{2} 0.34\right]$, but the extra magnetic Bragg peaks given by that propagation vector combined with the Gaussian line shape used for an individual peak make the final calculation a reasonable approximation for the scattering from a two-dimensional structure.

Care clearly must be taken in interpreting data from powdered samples of MPS ${ }_{3}$, as discussed in our recent article on $\mathrm{NiPS}_{3}$ [15]. Kurosawa et al. originally proposed the propagation vector $\mathbf{k}_{M}=\left[01 \frac{1}{2}\right]$ from measurements of single crystals that had been stacked with a common $\mathbf{c}^{\star}$ axis [10]. While they did not show their diffraction patterns, it seems reasonable that they did not see any magnetic intensity at (010). Intensity at this position appears to be purely an artifact of a powdered sample.

It is interesting to note that similar two-dimensional line shapes are observed in the powder diffraction for $\mathrm{MnPS}_{3}$ [37], which is another excellent example of a two-dimensional antiferromagnet [32,38], but not so much in $\mathrm{NiPS}_{3}$ [15]. While further studies on the magnetic dynamics of $\mathrm{NiPS}_{3}$ are ongoing, it is possible that this compound is not as good an approximation of a two-dimensional system as its sister compounds.

Comparison of the calculations for the magnon dynamics and the neutron data show some discrepancies. The discrepancies depend heavily on the magnitudes of the third-nearestneighbor exchange, $J_{3}$, and the anisotropy, $\Delta$, which hints at their source. The magnitudes for $J_{3}$ and $\Delta$ are large. The Hamiltonian used to model the data was very simple, assuming a Heisenberg model with a single-ion anisotropy. 


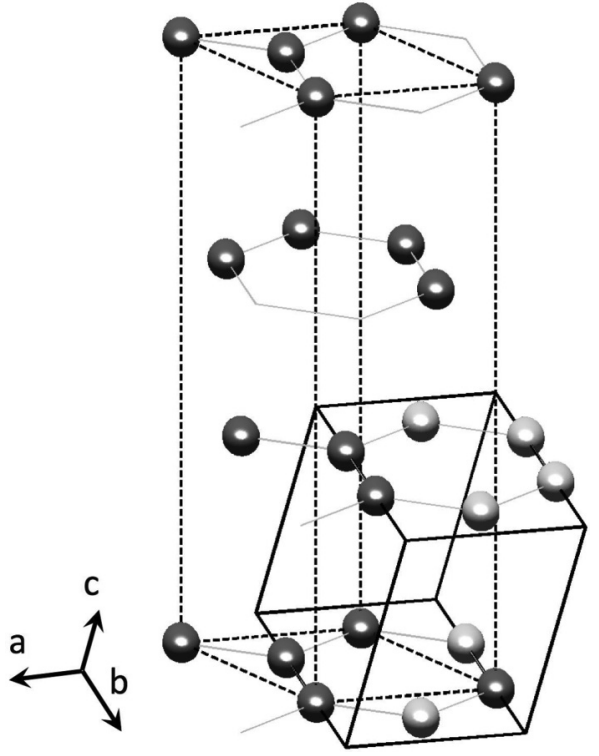

FIG. 10. Schematic showing the relationship between the monoclinic and orthohexagonal unit cells for $\mathrm{FePS}_{3}$. The positions of the $\mathrm{Fe}^{2+}$ positions are shown as spheres. Dark gray spheres represent ions that are in the hexagonal unit cell, while light gray spheres are only in the monoclinic unit cell.

It may be that, to an extent, the parameters $J_{3}$ and $\Delta$ are compensating for effects that are not accounted for in the Hamiltonian.

The calculation made numerous assumptions. Only the spin degrees of freedom were considered. Orbital contributions are known to be a factor in $\mathrm{FePS}_{3}$ [11] and are likely to impact on the treatment of the anisotropy. The interactions were taken to be short-ranged. Longer-ranged interactions, such as dipole-dipole effects [39,40], may play a role. The exchange parameters were considered to be equivalent for equivalent distances between moments, despite each moment being ferromagnetically coupled to two of its nearest neighbors and antiferromagnetically coupled to the third. Allowing for inequivalent exchange interactions between equivalent neighbors might be a consideration. This may be justified as the magnetic phase transition is believed to be first order, with a concomitant distortion of the lattice at $T_{N}$ as observed in $\mathrm{x}$-ray diffraction $[18,41]$.

However, the simple Hamiltonian in Eq. (1) does appear to capture the essential aspects of the magnetic dynamic structure factor. As previously observed [14], the values for the exchange interactions are consistent with the stability conditions for the in-plane magnetic structure as calculated for the classical magnetic phase diagram of a two-dimensional honeycomb lattice [42]. The ratios $J_{2} / J_{1}=-0.03$ and $J_{3} / J_{1}=-0.66$ give rise to a structure with ferromagnetic chains that are antiferromagnetically coupled, as is the case for $\mathrm{FePS}_{3}$. The values listed in Table IV may, therefore, be considered to be the best estimates for the magnetic exchange parameters in $\mathrm{FePS}_{3}$.

Further analysis of the neutron data using more complicated Hamiltonians will be considered in future work.
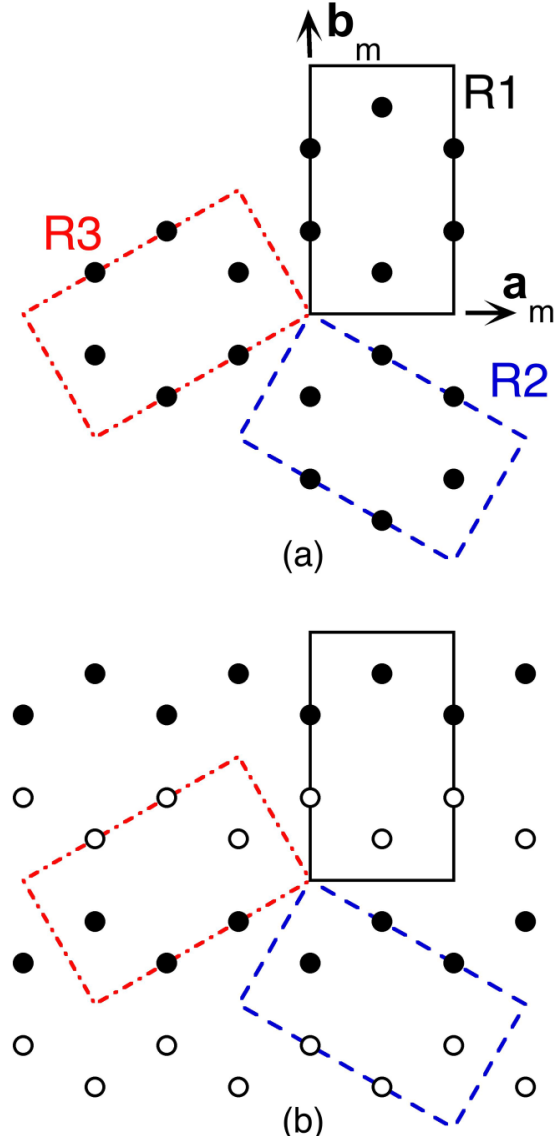

FIG. 11. (a) Schematic showing the $a b$ plane with the three twinned crystalline domains for the monoclinic cell. The points mark the positions for the $\mathrm{Fe}^{2+}$ ions. (b) The magnetic structure with an in-plane propagation vector $\mathbf{k}_{M}=(01)$ is drawn with respect to domain $R 1$. Open circles represent a moment out of the page and closed circles represent moments into the page. The other two domains are also shown.

\section{CONCLUSIONS}

The magnetic structure of $\mathrm{FePS}_{3}$ has been determined from neutron single-crystal diffraction and the magnetic propagation vector is $\mathbf{k}_{M}=\left[01 \frac{1}{2}\right]$. The magnon dynamics have been measured with neutron inelastic scattering. The magnetic exchange parameters, based on a simple Heisenberg Hamiltonian with a single-ion anisotropy, have been determined. They are consistent with previous measurements and with stability conditions, and they show that $\mathrm{FePS}_{3}$ is a very good example of a two-dimensional magnetic system.

\section{ACKNOWLEDGMENTS}

The authors thank the Institut Laue-Langevin, the ISIS facility, and Australian Nuclear Science and Technology Organisation for the use of their neutron instrumentation, and particularly the instrument teams on TAIPAN, MERLIN, D10, IN3, IN8 and IN20. A.R.W. thanks Dr. Elisa Wheeler, Dr. Juan Rodriguez-Caraval, Dr. Navid Qureshi, Professor Toby Perring, and Dr. Gøran Nilsen for helpful discussions and Wayne Clancy for his technical assistance in the fabrication 
TABLE V. Table with the Bragg peaks indexed from a previous study on a twinned crystal, along with alternative indexing using Eqs. (A2) and (A3).

\begin{tabular}{|c|c|c|}
\hline $\begin{array}{l}h k l \\
\text { from Ref. [12] }\end{array}$ & $\begin{array}{l}\text { Rotation operations } \\
\quad \text { (cf. Fig. 11) }\end{array}$ & $h k l$ \\
\hline$\left(\frac{3}{2} \frac{1}{2} \overline{0.66)}\right.$ & $R 2+180^{\circ}$ & $\left(1 \overline{2} \frac{\overline{1}}{2}\right)$ \\
\hline$\left(\frac{3}{2} \frac{1}{2} \overline{0.34}\right)$ & $R 2$ & $\left(\overline{1} 2 \frac{1}{2}\right)$ \\
\hline$\left(\frac{3}{2} \frac{1}{2} 0.34\right)$ & $R 2+180^{\circ}$ & $\left(1 \overline{2} \frac{1}{2}\right)$ \\
\hline$\left(\frac{3}{2} \frac{1}{2} 0.66\right)$ & $R 2$ & $\left(\overline{1} 2 \frac{1}{2}\right)$ \\
\hline$\left(\overline{\frac{3}{2} \frac{7}{2}} 0.34\right)$ & $R 3+180^{\circ}$ & $\left(1 \overline{4} \frac{1}{2}\right)$ \\
\hline$\left(\frac{1}{2} \frac{1}{2} \overline{0.66}\right)$ & $R 2$ & $\left(0 \overline{1} \frac{1}{2}\right)$ \\
\hline$\left(\frac{1}{2} \overline{\frac{11}{2}} \overline{0.66}\right)$ & $R 3$ & $\left(\overline{3} 2 \frac{1}{2}\right)$ \\
\hline$\left(\frac{1}{2} \frac{1}{2} 0.34\right)$ & $R 2$ & $\left(01 \frac{1}{2}\right)$ \\
\hline$\left(\frac{1}{2} \frac{\overline{11}}{2} 0.34\right)$ & $R 3$ & $\left(\overline{3} 2 \frac{3}{2}\right)$ \\
\hline$\left(\overline{\frac{1}{2}} \frac{5}{2} 0.34\right)$ & $R 3$ & $\left(\overline{1} 2 \frac{1}{2}\right)$ \\
\hline$\left(\frac{1}{2} \frac{\overline{7}}{2} \overline{0.34}\right)$ & $R 3$ & $\left(\overline{2} 1 \frac{1}{2}\right)$ \\
\hline$\left(\frac{1}{2} \frac{\overline{3}}{2} \overline{0.34}\right)$ & - & - \\
\hline$\left(\frac{1}{2} \frac{1}{2} \overline{0.66}\right)$ & $R 2$ & $\left(01 \overline{\frac{1}{2}}\right)$ \\
\hline$\left(\frac{3}{2} \frac{1}{2} \overline{0.66}\right)$ & $R 3+180^{\circ}$ & $\left(12 \overline{\frac{1}{2}}\right)$ \\
\hline$\left(\frac{3}{2} \frac{1}{2} \overline{1.34}\right)$ & $R 2$ & $\left(\overline{1} 2 \frac{1}{2}\right)$ \\
\hline$\left(\frac{1}{2} \frac{1}{2} \overline{0.66}\right)$ & $R 3$ & $\left(0 \overline{1} \frac{\overline{1}}{2}\right)$ \\
\hline$\left(\frac{5}{2} \frac{1}{2} \overline{0.66}\right)$ & $R 2$ & $\left(\overline{1} 4 \frac{1}{2}\right)$ \\
\hline$\left(\frac{5}{2} \frac{1}{2} \overline{1.66}\right)$ & $R 2$ & $\left(\overline{1} 4 \overline{\frac{1}{2}}\right)$ \\
\hline$\left(\frac{5}{2} \frac{1}{2} \overline{0.66}\right)$ & $R 3$ & $\left(\overline{14} \frac{1}{2}\right)$ \\
\hline
\end{tabular}

of the sample mount. Part of this work was performed while A.R.W. was on sabbatical at Australian Nuclear Science and Technology Organisation (ANSTO) and he thanks ANSTO for the financial support.

\section{APPENDIX: INDEXING IN THE PRESENCE OF TWINNED DOMAINS}

Crystal twinning is an important issue in $\mathrm{MPS}_{3}$ compounds. While the structural space groups are all monoclinic, the cell parameters are such that the structure is only slightly distorted from being orthohexagonal [3]. This is particularly relevant for $\mathrm{FePS}_{3}$ whose Bragg peaks can be equally indexed using either basis [3]. This ability derives from the fact that the relationships

$$
\begin{gathered}
b=\sqrt{3} a \\
a=-3 c \cos \beta
\end{gathered}
$$

hold to a high degree of precision in this compound. Figure 10 shows a schematic with the relationship between the two unit cells.
The lamellar structure of the compound is highly susceptible to stacking faults [43]. This, combined with the proximity of the two structures, explains the high probability that a crystal may be twinned with multiple domains rotated by $120^{\circ}$. The presence of these domains has been discussed at length in a recent article by Murayama et al. [18]. Figure 11(a) shows the arrangement of $\mathrm{Fe}^{2+}$ ions in the $a b$ plane. The three possible in-plane orientations for the monoclinic twinned domains are also shown. The direction of the $c$ axis, and thus the manner that the $a b$ planes stack, define the difference between the domains.

The three reciprocal lattices are linked by the rotation matrices:

$$
\begin{aligned}
{\left[\begin{array}{l}
h \\
k \\
l
\end{array}\right]^{R 1}=} & {\left[\begin{array}{rrr}
-1 / 2 & 1 / 2 & 0 \\
-3 / 2 & -1 / 2 & 0 \\
1 / 2 & -1 / 6 & 1
\end{array}\right]\left[\begin{array}{l}
h \\
k \\
l
\end{array}\right]^{R 2} } \\
= & {\left[\begin{array}{rrr}
-1 / 2 & -1 / 2 & 0 \\
3 / 2 & -1 / 2 & 0 \\
1 / 2 & 1 / 6 & 1
\end{array}\right]\left[\begin{array}{l}
h \\
k \\
l
\end{array}\right]^{R 3}, }
\end{aligned}
$$

where the superscript refers to the relevant orientation in Fig. 11. The application of these matrices shows that the (010) Bragg peak in domain $R 1$ will be indexed as $\left(\overline{\frac{1}{2}} \frac{1}{2} \frac{1}{6}\right)$ in domain $R 2$ and $\left(\frac{1}{2} \frac{1}{2} \frac{1}{6}\right)$ in domain $R 3$.

Figure 11(b) shows how the propagation vector may be confused if the magnetic Bragg peaks are indexed using the wrong domain. The in-plane propagation vector $\mathbf{k}_{M}=[01]$ is shown with respect to domain $R 1$. However, the propagation vector will appear as $\mathbf{k}_{M}=\left[\frac{1}{2} \frac{1}{2}\right]$ if domain $R 2$ is used to index the magnetic Bragg peaks and as $\mathbf{k}_{M}=\left[\frac{1}{2} \frac{1}{2}\right]$ if domain $R 3$ is used.

If the $l$ indices for the magnetic Bragg peaks listed in Ref. [12] are rounded to the nearest third, Eq. (A2) combined with the symmetry elements for the magnetic structure can reindex almost all of the peaks to an allowed reflection with a propagation vector of $\mathbf{k}_{M}=\left[01 \frac{1}{2}\right]$. The indexing sometimes requires an additional rotation by $180^{\circ}$ about the $\mathbf{c}^{\star}$ axis, which may be performed using the rotation matrix:

$$
\left[\begin{array}{l}
h \\
k \\
l
\end{array}\right]^{180^{\circ}}=\left[\begin{array}{rrr}
-1 & 0 & 0 \\
0 & -1 & 0 \\
2 / 3 & 0 & 1
\end{array}\right]\left[\begin{array}{l}
h \\
k \\
l
\end{array}\right] .
$$

The Bragg peaks from Fig. 3 and Table II in Ref. [12] and their new indices after rotation are listed in Table V. The only Bragg peak that could not be mapped onto an allowed peak for $\mathbf{k}_{M}=\left[01 \frac{1}{2}\right]$ was $\left(\frac{1}{2} \frac{3}{2} \overline{0.34}\right)$. This Bragg peak was specifically searched for during our D10 experiment in all possible domains, and no peak was detected. It seems highly likely that this Bragg peak was spurious in the previous experiment.
[1] V. Grasso and L. Silipigni, Riv. Nuovo Cimento 25(6), 1 (2002).

[2] R. Brec, Solid State Ionics 22, 3 (1986).
[3] G. Ouvrard, R. Brec, and J. Rouxel, Mater. Res. Bull. 20, 1181 (1985). 
[4] I. Tyagur, Func. Mater. Lett. 2, 95 (2009).

[5] D. Yang, P. Westreich, and R. F. Frindt, J. Solid State Chem. 166, 421 (2002).

[6] R. F. Frindt, D. Yang, and P. Westreich, J. Mater. Res. 20, 1107 (2005).

[7] K. Du, X. Wang, Y. Liu, P. Hu, M. I. B. Utama, C. K. Gan, Q. Xiong, and C. Kloc, ACS Nano 10, 1738 (2016).

[8] C.-T. Kuo, M. Neumann, K. Balamurugan, H. J. Park, S. Kang, H. W. Shiu, J. H. Kang, B. H. Hong, M. Han, T. W. Noh et al., Sci. Rep. 6, 20904 (2016).

[9] J. G. Park, J. Phys.: Condens. Matter 28, 301001 (2016).

[10] K. Kurosawa, S. Saito, and Y. Yamaguchi, J. Phys. Soc. Japan 52, 3919 (1983).

[11] P. A. Joy and S. Vasudevan, Phys. Rev. B 46, 5425 (1992).

[12] K. C. Rule, G. J. McIntyre, S. J. Kennedy, and T. J. Hicks, Phys. Rev. B 76, 134402 (2007).

[13] Note that Kurosawa et al. quote a magnetic propagation vector of $\mathbf{k}_{M}=\left[00 \frac{1}{2}\right]$ in their paper. However, this is in error and the correct propagation vector for the structure they propose is $\mathbf{k}_{M}=\left[01 \frac{1}{2}\right]$.

[14] A. R. Wildes, K. C. Rule, R. I. Bewley, M. Enderle, and T. J. Hicks, J. Phys.: Condens. Matter 24, 416004 (2012).

[15] A. R. Wildes, V. Simonet, E. Ressouche, G. J. McIntyre, M. Avdeev, E. Suard, S. A. J. Kimber, D. Lançon, G. Pepe, B. Moubaraki et al., Phys. Rev. B 92, 224408 (2015).

[16] J. R. Stewart, P. P. Deen, K. H. Andersen, H. Schober, J.-F. Barthélémy, J. M. Hillier, A. P. Murani, T. Hayes, and B. Lindenau, J. Appl. Cryst. 42, 69 (2009).

[17] C. M. E. Zeyen, R. Chagnon, F. Disdier, and H. Morin, Revue Phys. Appl. 19, 789 (1984).

[18] C. Murayama, M. Okabe, D. Urushihara, T. Asaka, K. Fukuda, M. Isobe, K. Yamamoto, and Y. Matsushita, J. Appl. Phys. 120, 142114 (2016).

[19] C. Wilkinson, H. W. Khamis, R. F. D. Stansfield, and G. J. McIntyre, J. Appl. Crystallogr. 21, 471 (1988).

[20] J. Rodriguez-Carvajal, Physica B 192, 55 (1993).

[21] S. A. Danilkin, M. Yethiraj, T. Saerbeck, F. Klose, C. Ulrich, J. Fujioka, S. Miyasaka, Y. Tokura, and B. Keimer, J. Phys.: Conf. Ser. 340, 012003 (2012).

[22] A. Wildes, T. J. Hicks, D. Lançon, A. Magrez, H. Rønnow, K. Rule, and H. Walker (2014), doi:10.5291/ILL-DATA.4-01-1409.

[23] A. Wildes, T. J. Hicks, D. Lançon, A. Piovano, H. Rønnow, K. Rule, and H. Walker (2016), doi:10.5291/ILL-DATA.4-01-1513.
[24] R. I. Bewley, R. S. Eccleston, K. A. McEwen, S. M. Hayden, M. T. Dove, S. M. Bennington, J. R. Treadgold, and R. L. S. Coleman, Physica B 385-386, 1029 (2006).

[25] http://www.bellexinternational.com/products/cytop/, accessed 2016-09-30.

[26] H. A. Mook, P. Dai, S. M. Hayden, G. Aeppli, T. G. Perring, and F. Doğan, Nature 395, 580 (1998).

[27] T. Huberman, R. Coldea, R. A. Cowley, D. A. Tennant, R. L. Leheny, R. J. Christianson, and C. D. Frost, Phys. Rev. B 72, 014413 (2005).

[28] N. B. Christensen, H. M. Rønnow, D. F. McMorrow, A. Harrison, T. G. Perring, M. Enderle, R. Coldea, L. P. Regnault, and G. Aeppli, Proc. Natl. Acad. Sci. USA 104, 15264 (2007).

[29] M. Russina and F. Mezei, Nuc. Instrum. Meth. Phys. Res. Sect. A 604, 624 (2009)

[30] M. Nakamura, R. Kajimoto, Y. Inamura, F. Mizuno, M. Fujita, T. Yokoo, and M. Arai, J. Phys. Soc. Japan 78, 093002 (2009).

[31] K. Momma and F. Izumi, J. Appl. Crystallogr. 44, 1272 (2011).

[32] A. R. Wildes, H. M. Rønnow, B. Roessli, M. J. Harris, and K. W. Godfrey, Phys. Rev. B 74, 094422 (2006).

[33] E. M. Wheeler, R. Coldea, E. Wawrzyńska, T. Sörgel, M. Jansen, M. M. Koza, J. Taylor, P. Adroguer, and N. Shannon, Phys. Rev. B 79, 104421 (2009).

[34] O. Arnold, J. C. Bilheux, J. M. Borreguero, A. Buts, S. I. Campbell, L. Chapon, M. Doucet, N. Draper, R. Ferraz Leal, M. A. Gigg et al., Nuc. Instrum. Meth. Phys. Res. Sect. A 764, 156 (2014)

[35] R. A. Ewings, A. Buts, M. D. Le, J. van Duijn, I. Butinduy, and T. G. Perring, Nucl. Instrum. Methods. Phys. Res. A 834, 132 (2016).

[36] K. C. Rule, T. Ersez, S. J. Kennedy, and T. J. Hicks, Physica B 335, 6 (2003)

[37] A. R. Wildes, S. J. Kennedy, and T. J. Hicks, J. Phys: Condens. Matter 6, L335 (1994).

[38] A. R. Wildes, B. Roessli, B. Lebech, and K. W. Godfrey, J. Phys: Condens. Matter 10, 6417 (1998).

[39] C. Pich and F. Schwabl, Phys. Rev. B 47, 7957 (1993).

[40] C. Pich and F. Schwabl, J. Magn. Magn. Mater. 148, 30 (1995).

[41] P. Jernberg, B. Bjarman, and R. Wäppling, J. Magn. Magn. Mater. 46, 178 (1984).

[42] J. B. Fouet, P. Sindzingre, and C. Lhuillier, Eur. Phys. J. B. 20 , 241 (2001).

[43] G. Ouvrard and R. Brec, Eur. J. Solid State Inorg. Chem. 27, 477 (1990). 